\title{
Stator Winding Fault Detection Using External Search Coil and Artificial Neural Network
}

\author{
João Vicente ${ }^{1,2}$, Angela Ferreira $^{2^{*}}$, Marcelo Castoldi ${ }^{1}$, João Teixeira ${ }^{2}$, \\ and Alessandro Goedtel ${ }^{1}$ \\ ${ }^{1}$ Federal Technological University of Paraná, Cornélio Procópio, Paraná, Brazil \\ ${ }^{2}$ Research Centre in Digitalization and Intelligent Robotics (CeDRI), Instituto Politécnico de \\ Bragança, Portugal
}

\begin{abstract}
This paper presents a methodology for winding stator fault detection of induction motors, using an external search coil, which is a noninvasive technique and can be applied during motor operation. The dispersion magnetic flux of the motor operating in abnormal conditions induces a voltage in the search coil that differs from a reference pattern corresponding to the healthy stator winding. Experimental data were obtained in a test bench using a $0.75 \mathrm{~kW}$ three-phase squirrel-cage induction motor with the stator winding modified to allow the introduction of short circuits. This work considered short circuits in one phase, involving $1 \%, 3 \%$, $5 \%$ and $10 \%$ of the turns, with the motor loaded with a varying torque. Fault diagnosis is obtained through two models of artificial neural networks, implemented with the signals in the time domain. The obtained results demonstrated that the developed methodology presents difficulties in predicting short circuits in incipient stages, but for short circuits of higher severity, the behaviour improved substantially, being $100 \%$ successful for faults with $10 \%$ turns short-circuited.
\end{abstract}

\section{Introduction}

Squirrel cage induction motors are widely used in the industrial sector, due to low-cost and low maintenance requirements, high reliability and easy adaptation to various mechanical loads. However, despite their robustness and reliability, induction motors are subjected to electrical or mechanical failures, which occurs due to prolonged and severe operating conditions such as overload, overvoltage, voltage and current unbalance, among others [1$3]$.

According to [4], the major faults in induction motors occurs in bearings (69\%), stator winding $(21 \%)$, rotor bars $(7 \%)$, with $3 \%$ probability of occurrence in other parts. Another study [5] suggests that the probability of occurrence of faults due to bearings failure is about $40 \%$, while stator and rotor windings failures are about $38 \%$ and $10 \%$, respectively.

Faults related with stator windings are typically due to insulation stress, which may cause inter-turns short-circuits, phase-to-phase and/or single phase to ground short-circuits.

\footnotetext{
*Corresponding author: apf@ipb.pt
} 
In the presence of a short-circuit involving some turns, the temperature will rise and, in consequence, insulation of adjacent turns will deteriorate and may cause irreversible damage to the machine $[6,7]$. The fault detection in an incipient stage enables the reduction of the repair costs and the interruption of the production process. Hence, fault diagnosis in induction motors (IM) is of major importance as part of predictive maintenance.

Several quantities in the machine are susceptible to suffer changes in the presence of a fault as, for instance, temperature, vibration, magnetic flux, current, among others, and are frequently used to diagnose a fault. The work reported in [8] presents the stator winding fault diagnosis through the spectral frequency analysis of the magnetic flux and also vibration. Another approach, presented in [5], involves the detection of short-circuits in the stator winding by analysing the symmetrical components of the stator currents. The monitoring of inter-turn short circuits of IM stator windings under direct field-oriented control is introduced in [9], by the spectral analysis of internal signals of the control structure.

More recently, several approaches based on intelligent systems, such as artificial neural networks (ANN), fuzzy logic, genetic algorithms, decision trees, among others, have been used in fault diagnosis of three-phase IM, using the learning ability by extraction of characteristics involving the process in study. Intelligent systems based tools do not involve complex mathematic models, which in turn favours the reduction of the computation complexity in the implementation and application phases [10, 11].

The authors in [1] utilize ANN and decision trees with delayed stator current signals for fault detection in the stator windings, while [12] exploit fuzzy logic strategy with stator current Concordia patterns and [13] use an induction motor model, together with a genetic algorithm, exploiting stator voltages and currents and also mechanical speed.

Changes in the magnetic flux due to faults can be detected by search coils, placed inside the machine, in the stator slots, or externally, the latter having the advantage of being a nonintrusive approach. The work presented in [14] uses an external search coil, from which the induced electromotive force is analysed through a frequency spectral approach for broken bars detection. In [6] stator winding fault diagnosis is implemented with an internal observer coil, by total harmonic distortion analysis compared to the healthy condition.

This work addresses the stator winding fault diagnosis of a three-phase IM using an external search coil, which is a non-invasive technique and can be applied to operating machines. The dispersion magnetic flux of the motor operating in abnormal conditions induces a voltage in the exploratory coil that differs from a reference pattern corresponding to the healthy stator winding. The data processing is exploited in the time domain and failure severity classification is achieved via an ANN.

The work in this paper has been structured as follows: section 2 presents a brief theoretical foundation on artificial neural networks; in section 3 the experimental approach is detailed and the search coil system is presented. The experimental results are presented in section 4 , and finally, the conclusions are addressed in section 5.

\section{Artificial neural networks}

Artificial neural networks are computational models inspired on the nervous system of living beings, formed by a set of processing units called neurons, interconnected each other between layers, through artificial synapses $[15,16]$. The peculiarity of the neural network is the learning ability from experience, adapting to changing inputs and generating results without redesigning the output criteria.

The artificial neurons have activation functions that limit their output. Some activation functions used are tangent hyperbolic (tansig), linear (purelin), logistic (logsig), and elliot symmetric sigmoid (elliotsig) [17]. 
There are several types of topologies for ANN, being the most common the multilayer perceptron (MLP) [1]. This topology has at least one hidden layer, between input and output layers and the same activation function of neurons in the same layer [15, 18]. Fig. 1 shows the topology multilayer perceptron ANN, formed by one hidden layer. The process of adjusting the ANN parameters to acquire knowledge is called training. The training of the multilayer perceptron topology uses a backpropagation algorithm, which occurs in two stages. The backpropagation algorithm is based on the descendent gradient that requires the use of derivable activation functions. In the first stage, the training data is applied in the input layer of the network and the results are obtained in the output layer, being compared with the targets, generating an error. In a second stage, the parameters of the ANN are adjusted, based on the error obtained in the last stage. The process is applied in successive iterations with the objective of minimizing the error between the network output and targets $[1,16]$.

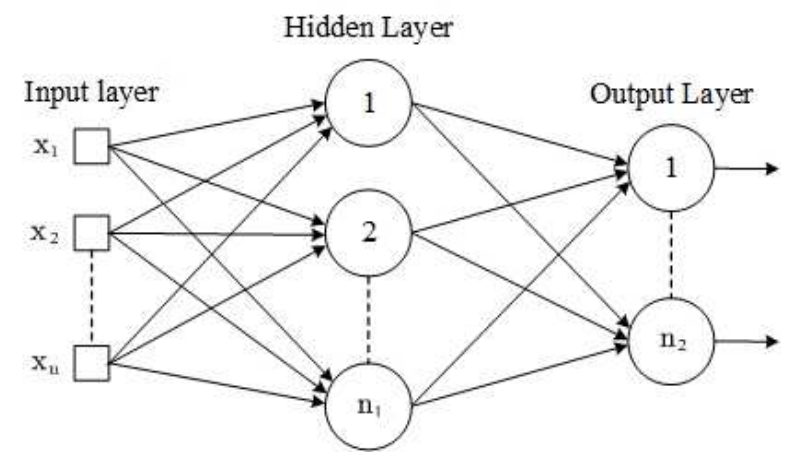

Fig. 1. Artificial neural network multilayer perceptron topology.

Variations and optimization of the backpropagation training algorithm arose with the objective of reducing computational costs, optimizing results and search besides local minimums. Among them, there are the Training Levenberg-Marquardt backpropagation [19, 20] (trainlm), Training Bayesian Regularization backpropagation [21] (trainbr) and training resilient backpropagation [22] (trainrp).

The set of samples to be used in a neural network is divided into 3 sub-sets, one for training, another for validation, and finally, the third, for testing. The training set is used for the learning process. The validation set is used as a cross-validation set to avoid overfitting in the learning process. The test set is not used during the learning process and it is used only for final test purposes.

The cross-validation of the ANN allows estimating the capacity of generalization of topology to a new data set. The 10k-fold is a method of cross-validation that consists in the division of the data set in ten sets, from which nine sets are used for training and validation, and the last one is utilized for testing. In this method, a topology of the artificial neural network is trained ten times and tested ten times with a different test set and all samples are used exactly once as the test data [18].

\section{Experimental data acquisition}

\subsection{Work Bench}

The test bench to acquire the experimental data is shown in Fig. 2. Besides monitoring electrical and mechanical quantities, the experimental setup allows load torque variation of the IM through a DC generator with a resistive load, applying voltage unbalances and faults 
simulations in IM, such as broken bars in the rotor, short-circuit in stator windings, and bearing faults.

The tested three-phase squirrel-cage induction motor of $0.75 \mathrm{~kW}, 220 \mathrm{~V}$ delta connected, 4 poles, $60 \mathrm{~Hz}$, has a stator winding with 6 coils and 306 turns per phase, modified in such a way that it allows performing short circuit simulations of $1 \%, 3 \%, 5 \%$ and $10 \%$ of the turns of a phase.

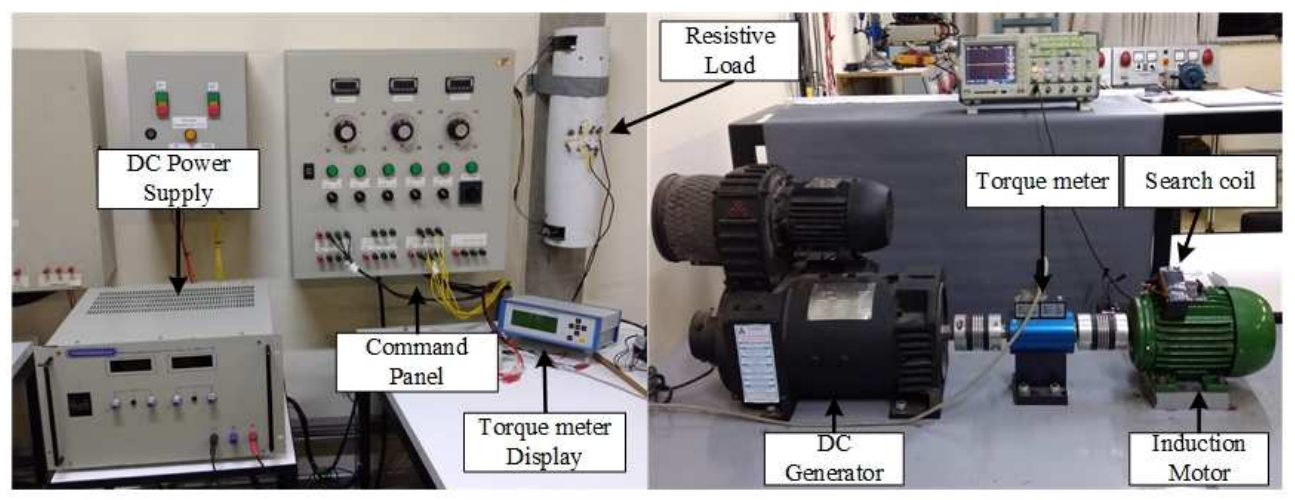

Fig. 2. Experimental test bench.

Several configurations of the external search coil have been proposed in the literature. For instance, in [23] there were tested three external coils with different core configurations (Fig. 3). The "U" core configuration presented better results in terms of the amplitude of the induced voltage and it had been adopted in this work. A simplified dimensioning approach led to the utilization of 291 turns in the search coil.
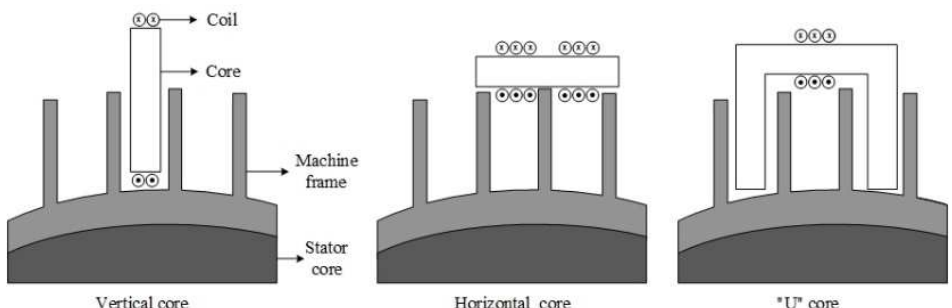

Fig. 3. External search coil configurations (adapted from [23]).

The optimal position of the search coil in the motor frame, i.e., the location of the search coil in order to maximize the amplitude of the induced voltage with minimum distortion of the induced signal, was defined through experimentation. It was found that the better position of the coil is in the central part of the motor frame.

\subsection{Proposed Fault Detection Method}

The methodology approach for fault detection on stator winding of an IM is presented schematically in Fig.4. 


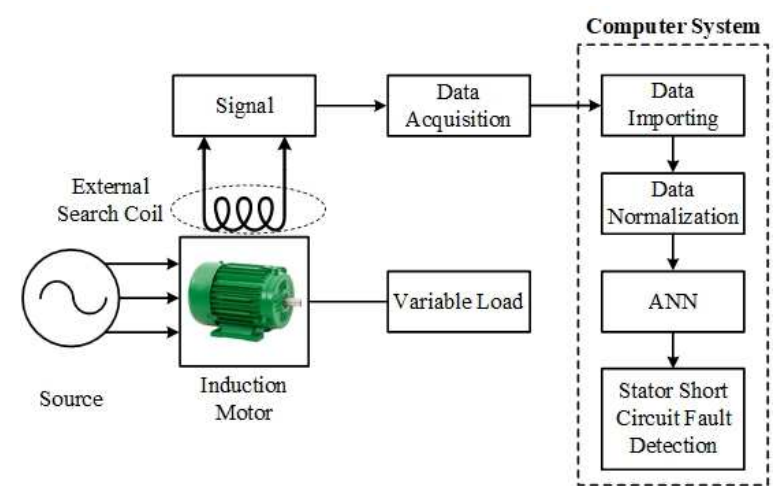

Fig. 4. Scheme of the proposed fault detection methodology.

The induced voltage signals were acquired through the oscilloscope TBS 1072b model from Tektronix, with a sampling frequency of $25 \mathrm{kHz}$ in 100 milliseconds. The signals were acquired for the healthy condition and faulty conditions of $1 \%, 3 \%, 5 \%$, and $10 \%$ turns shortcircuited in one phase of the stator winding. For each situation, the motor was loaded with a varying torque of $0,5 \mathrm{Nm}$ till $4,5 \mathrm{Nm}$ with steps of $0,5 \mathrm{Nm}$. The data generated have nine samples with six cycles each, for the five conditions identified previously.

The database was imported and processed using MATLAB. The fault diagnosis was implemented using neural networks developed under the guidelines proposed in Sect. 2, classifying the signals of the induced voltages in the time domain.

The data were subjected to a normalization process since the amplitudes of the induced voltage vary with the load torque and these fluctuations may influence the performance of the ANN. Main results of the studied ANN models are presented in the following section.

\section{Results and discussion}

Preliminary tests were implemented with 6 cycles each sample, but the results were not satisfactory because of the high number of input nodes (2500) and low number of samples (45) for training and test process. Therefore, each sample with 6 cycles was split into 6 samples with one cycle, assuming that one cycle contains information enough about the motor condition. This procedure allowed increasing the number of samples to 270 (54 samples for each operating condition) and reduce the input to 416 nodes. This situation is more suitable to train the ANN because it has less number of weights to tune and more samples for the learning process.

A feedforward multilayer perceptron topology was selected for the ANN, given the amount of the dataset available and the objective of classifying signals.

The selection of the architecture and hyper-parameters of the ANN were defined in a preliminary test, to select two ANN models (MLP1 and MPL2). Therefore, these models were trained and tested with 10k-fold method for all dataset.

The preliminary test has the objective of selecting the best train algorithm, number of neurons in the hidden layer and activation functions in hidden and output layers (HL and OL, respectively). Several combinations have been tested. The dataset was divided in $80 \%, 10 \%$ and $10 \%$ for train, validation and test sets, respectively, keeping the balance between classes in each set. Table 1 presents the accuracy in the test set for the ten best models.

Models 4, 5, 7, and 8 present higher accuracy. The best models with Bayesian Regularization (Trainbr) and Levenberg-Marquardt (Trainlm) were selected. Therefore, models 5 and 8 were selected and renamed as MLP1 and MLP2, respectively. 
Table 1. Preliminary test results.

\begin{tabular}{|c|c|c|c|c|c|}
\hline Model & Train Algor. & \# Neurons HL & HL Function & OL Function & Accuracy [\%] \\
\hline 1 & Trainbr & 45 & Tansig & Elliotsig & 66,7 \\
\hline 2 & Trainbr & 30 & Elliotsig & Elliotsig & 70,4 \\
\hline 3 & Trainbr & 25 & Tansig & Purelin & 74,0 \\
\hline 4 & Trainbr & 25 & Elliotsig & Elliotsig & 85,2 \\
\hline 5 & Trainbr & 25 & Logsig & Elliotsig & 88,9 \\
\hline 6 & Trainlm & 25 & Tansig & Purelin & 77,8 \\
\hline 7 & Trainlm & 50 & Tansig & Elliotsig & 85,2 \\
\hline 8 & Trainlm & 35 & Tansig & Elliotsig & 85,2 \\
\hline 9 & Trainlm & 50 & Elliotsig & Elliotsig & 81,5 \\
\hline 10 & Trainlm & 25 & Elliotsig & Elliotsig & 81,5 \\
\hline
\end{tabular}

The two models MLP1 and MLP2 were trained with 10k-fold cross-validation procedure. Tables 2 and 3 present the confusion matrix for both models were target classes are in rows and predicted classes are in columns.

The last column of the confusion matrix gives the accuracy for each class, that is, for a given class, it states the rate of correct identifications performed by the network, and the last line presents the overall accuracy.

The overall accuracy of the structure MLP1 is $68,9 \%$. This low accuracy is due to the confusion that the network generated between the samples for healthy and $1 \%$ turns shortcircuited, classifying correctly $57,4 \%$ the cases healthy and $46,3 \%$ the later, while the $10 \%$ turns short-circuited were totally identified.

The overall accuracy with MLP2 is $68,5 \%$. Similar to what happened with MLP1, misclassified samples are present between the healthy and $1 \%$ turns short-circuited samples. The 3\% turns short-circuited situation was not classified correctly, presenting a low accuracy $(37,0 \%)$, however, the $10 \%$ turns short-circuited situation was classified correctly for all samples.

A second experiment was developed removing the faulty class corresponding to $1 \%$ turns short-circuited. The confusion matrixes for MLP1 and MLP2 with the remaining four classes are presented in Tables 4 and 5, respectively. MLP1 model increased the overall accuracy to $81,5 \%$, and the healthy class classification increased to up to $90,7 \%$. The higher misclassification happens now between $3 \%$ and 5\% turns short-circuited classes. The samples corresponding to $10 \%$ turns short-circuited were all correctly identified.

Table 2. Confusion matrix for the MLP1 with five classes.

\begin{tabular}{|c|c|c|c|c|c|c|}
\cline { 2 - 7 } \multicolumn{1}{c|}{} & $\mathrm{H}$ & $1 \%$ & $3 \%$ & $5 \%$ & $10 \%$ & $\begin{array}{c}\text { Acc. } \\
{[\%]}\end{array}$ \\
\hline $\mathrm{H}$ & 31 & 22 & 1 & 0 & 0 & 57,4 \\
\hline $1 \%$ & 19 & 25 & 10 & 0 & 0 & 46,3 \\
\hline $3 \%$ & 6 & 7 & 33 & 8 & 0 & 61,1 \\
\hline $5 \%$ & 1 & 1 & 9 & 43 & 0 & 79,6 \\
\hline $10 \%$ & 0 & 0 & 0 & 0 & 54 & 100 \\
\hline $\begin{array}{l}\text { Occ. } \\
{[\%]}\end{array}$ & 54,4 & 45,5 & 62,3 & 84,3 & 100 & $\mathbf{6 8 , 9}$ \\
\hline
\end{tabular}

Table 3. Confusion matrix for the MLP2 with five classes.

\begin{tabular}{|c|c|c|c|c|c|c|}
\cline { 2 - 7 } \multicolumn{1}{c|}{} & $\mathrm{H}$ & $1 \%$ & $3 \%$ & $5 \%$ & $10 \%$ & $\begin{array}{c}\text { Acc. } \\
{[\%]}\end{array}$ \\
\hline $\mathrm{H}$ & 38 & 15 & 0 & 1 & 0 & 70,4 \\
\hline $1 \%$ & 16 & 29 & 8 & 1 & 0 & 53,7 \\
\hline $3 \%$ & 11 & 8 & 20 & 15 & 0 & 37,0 \\
\hline $5 \%$ & 1 & 2 & 7 & 44 & 0 & 81,5 \\
\hline $10 \%$ & 0 & 0 & 0 & 0 & 54 & 100 \\
\hline $\begin{array}{l}\text { Occ. } \\
{[\%]}\end{array}$ & 57,6 & 53,7 & 57,1 & 72,1 & 100 & $\mathbf{6 8 , 5}$ \\
\hline
\end{tabular}


Table 4. Confusion matrix for the MLP1 neural network with four classes.

\begin{tabular}{|c|c|c|c|c|c|}
\cline { 2 - 6 } \multicolumn{1}{c|}{} & $\mathrm{H}$ & $3 \%$ & $5 \%$ & $10 \%$ & $\begin{array}{c}\text { Acc. } \\
{[\%]}\end{array}$ \\
\hline $\mathrm{H}$ & 49 & 5 & 0 & 0 & 90,7 \\
\hline $3 \%$ & 9 & 33 & 12 & 0 & 61,1 \\
\hline $5 \%$ & 1 & 13 & 40 & 0 & 74,1 \\
\hline $10 \%$ & 0 & 0 & 0 & 54 & 100 \\
\hline $\begin{array}{l}\text { Occ. } \\
{[\%]}\end{array}$ & 83,1 & 64,7 & 76,9 & 100 & $\mathbf{8 1 , 5}$ \\
\hline
\end{tabular}

Table 5. Confusion matrix for the MLP2 neural network with four classes.

\begin{tabular}{|c|c|c|c|c|c|}
\cline { 2 - 6 } \multicolumn{1}{c|}{} & $\mathrm{H}$ & $3 \%$ & $5 \%$ & $10 \%$ & $\begin{array}{c}\text { Acc. } \\
{[\%]}\end{array}$ \\
\hline $\mathrm{H}$ & 51 & 3 & 0 & 0 & 94,4 \\
\hline $3 \%$ & 9 & 36 & 9 & 0 & 66,7 \\
\hline $5 \%$ & 3 & 6 & 45 & 0 & 83,3 \\
\hline $10 \%$ & 0 & 0 & 0 & 54 & 100 \\
\hline $\begin{array}{l}\text { Occ. } \\
{[\%]}\end{array}$ & 81,0 & 80,0 & 83,3 & 100 & $\mathbf{8 6 , 1}$ \\
\hline
\end{tabular}

Regarding MLP2 model with the four classes under analysis, the overall accuracy increased to $86,1 \%$. There was an increase in the detection rate for the healthy signature. Similar to the structure MLP1, the network presented small confusions between the faulty situations corresponding to $3 \%$ and 5\% turns short-circuited. Again, the $10 \%$ turns shortcircuited samples were all correctly identified.

With the exclusion of $1 \%$ fault samples from the database, the accuracy of the two models increased substantially, presenting improved accuracy in the classification of motor operating conditions, mainly for healthy motor samples. Failures corresponding to $10 \%$ turns shortcircuited were all identified in all experiments presented.

Based on the higher accuracy, the best model tested was MLP2 for the four classes. Even with confusion between the $3 \%$ and $5 \%$ failures, the model identified that the motor was running in healthy operation in $94,4 \%$ of the cases. For all the samples involving failures, the developed network was able to classify $92,6 \%$ of the cases with some type of failure, even if the severity was misclassified.

\section{Conclusions and future work}

This work proposed a methodology for the detection of winding stator faults of induction motors, based in the time domain analysis of the induced voltage signal in an external search coil by means of ANN. The results obtained used real data, generated experimentally from healthy and faulty conditions of an IM, able to simulate the functioning in an industrial environment.

The results obtained present misclassification of healthy and 1\% turns short-circuited operation modes, with an overall accuracy of about $69 \%$. This output may suggest that the dimensioning of the search coil should be reviewed, in order to improve the detection of faulty conditions in incipient stages. However, removing the data of faults involving $1 \%$ turns, one of the structures under study (MLP2) presented an accuracy above $86 \%$, and the healthy operation identification reached $94 \%$. It is worth noting that for the most significant faulty condition under consideration (10\% turns short-circuited) all tested configurations were $100 \%$ successful.

From the above, the methodology proposed in this work presented difficulties in predicting short circuits in incipient stages, but for short circuits of higher severity, the behaviour improved substantially.

Future work should explore the frequency analysis of the data acquired, in order to test the best approach. It is also suggested resize the search coil in order to improve the signal 
differentiation at early stages of faulty conditions. The dataset should also be increased, by testing other IM, with increased rated power.

\section{Acknowledgment}

This work has been supported by FCT - Fundação para a Ciência e Tecnologia within the Project Scope: UIDB/05757/2020.

\section{References}

1. G. H. Bazan, P. R. Scalassara, W. Endo, A. Goedtel, R. H. C. Palacios, W. F. Godoy, IEEE Trans. Ind. Electron. 66, 4, 3237-3246 (2019)

2. R. H. Palacios, I. N. Da Silva, A. Goedtel, W. F. Godoy, T. D. Lopes, IEEE Trans. Ind. Informatics 13, 4,1681-1691 (2017)

3. R. Sadeghi, H. Samet, T. Ghanbari, IEEE Trans. Ind. Informatics 15, 8, 4506-4515, (2019)

4. A. Bellini, F. Filippetti, C. Tassoni, G. A. Capolino, IEEE Trans. Ind. Electron. 55, 12, 4109-4126 (2008)

5. M. B. K. Bouzid, G. Champenois, IEEE Trans. Ind. Electron. 60, 9, 4093-4102 (2013).

6. G. N. Surya, Z. J. Khan, M. S. Ballal, H. M. Suryawanshi, IEEE Trans. Ind. Electron. 64, 2, 1495-1506 (2017)

7. M. Riera-Guasp, J. A. Antonino-Daviu, G. A. Capolino, IEEE Trans. Ind. Electron. 62, 3, 1746-1759 (2015)

8. P. C. M. L. Filho, R. Pederiva, J. N. Brito, Mech. Syst. Signal Process. 42, 1-2, 377-387 (2014)

9. M. Wolkiewicz, G. Tarchała, T. Orłowska-Kowalska, C. T. Kowalski, IEEE Trans. Ind. Electron. 63, 4, 2517-2528 (2016)

10. R. H. Cunha Palácios, I. N. Da Silva, A. Goedtel, W. F. Godoy, Electr. Power Syst. Res. 127, 249-258 (2015)

11. S. Haroun, A. N. Seghir, S. Touati, IET Electr. Power Appl. 12, 3, 339-346 (2018).

12. F. Zidani, M. E. H. Benbouzid, D. Diallo, M. S. Naït-Saïd, IEEE Trans. Energy Convers. 18, 4, 469-475 (2003)

13. M. Alamyal, S. M. Gadoue, B. Zahawi, Detection of induction machine winding faults using genetic algorithm (Proc. - 2013 9th IEEE Int. Symp. Diagnostics Electr. Mach. Power Electron. Drives, SDEMPED 2013, 157-161, 2013)

14. A. Bouzida, O. Touhami, R. Ibtiouen, Rotor fault diagnosis using external search coils voltage analysis (Proc. - 2014 Int. Conf. Electr. Mach. ICEM 2014, 1654-1658, 2014)

15. S. Haykin, Neural networks: a comprehensive foundation (Prentice Hall PTR, 2004)

16. H. L. Schmitt, P. R. Scalassara, A. Goedtel, W. Endo, J. Control. Autom. Electr. Syst. 26, 5, 535-544 (2015)

17. C. C. Aggarwal, Neural Networks and Deep Learning (Springer International Publishing, 2018)

18. I. N. Da Silva, D. H. Spatti, R. A. Flauzino, L. H. B. Liboni, S. F dos Reis Alves., Artificial neural networks: A practical course (Springer International Publishing, 2017)

19. K. Levenberg, Q.Appl. Math. 2, 164-168 (1944) 
20. D. Marquardt. An Algorithm for Least-Squares Estimation of Nonlinear Parameters. SIAM J. Appl. Math. 11, 2, 431-441 (1963)

21. D. J. C. MacKay, Neural Comput. 4, 3, 415-447 (1992)

22. M. Riedmiller, H. Braun, RPROP - A Fast Adaptive Learning Algorithm (Proceedings of the International Symposium on Computer and Information Science VII, 1992)

23. H. B. Ertan, O. Keysan, External search coil as a means of measuring rotor speed of an induction motor (8th Int. Symp. Adv. Electromechanical Motion Syst. Electr. Drives Jt. Symp. Electromotion, 1-6, 2009) 